Www.jmscr.igmpublication.org

Impact Factor (SJIF): 6.379

Index Copernicus Value: 79.54

ISSN (e)-2347-176x ISSN (p) 2455-0450

crossrefDOI: https://dx.doi.org/10.18535/jmscr/v6i9.159

Journal Of Medical Science And Clinical Research

\title{
Study of Recurrent Rhinosporidiosis in a Tertiary Care Centre of an Endemic Area
}

\author{
Authors \\ Dr Suneer R, Dr Sivasankari L, Dr Sivaram M \\ Kanyakumari Government Medical College, Asaripallam, Kanyakumari District, Tamil Nadu \\ Corresponding Author \\ Dr Sivasankari L \\ Associate Professor of ENT, Kanyakumari Government Medical College
}

\begin{abstract}
Background: Rhinosporidiosis is a chronic granulomatous inflammation caused by Rhinosporidium Seebri. It is a water borne disease endemic in Kanyakumari District of South India.

Objectives: To study the clinical profile of recurrent Rhinosporidiosis in an endemic area of Kanyakumari District, Tamilnadu.

Methods: This is a prospective study of 40 surgically treated cases of recurrent Rhinosporidiosis carried out in the Department of ENT, Kanyakumari Government Medical College from January 2016 to June 2017. All were diagnosed on clinical basis and were treated by excision and electrocautery of the base. The specimens were sent for histopathological examination.

Inclusion Criteria: Cases of Recurrent Rhinosporidiosis Willing for Surgery

Exclusion Criteria: Primary Rhinosporidiosis cases were excluded in this study.

Observation: The cases in this study were in the age group between 22 to 72 years. Of these 32 were males and 8 were females indicating a male preponderance. The main presenting symptoms was epistaxis in about $80 \%$ cases. Feeling of lump in the throat (8\%) is the second most common presenting symptom. Extra nasal spread is common in recurrent cases.

Conclusion: The study reflects the endemecity of this disease in Kanyakumari District of Tami Nadu. Extra Nasal Spread is common in recurrent disease. Special care in management of primary Rhinosporidiosis helps in preventing extranasal spread and recurrence of disease.

Keywords: Rhinosporidiosis, Recurrenc.
\end{abstract}

\section{Introduction}

Rhinosporidiosis is a chronic granulomatous inflammation commonly affecting the mucous membrane of nose, nasopharynx and lacrimal $\operatorname{sac}^{[1]}$. Other rare sites includes lips, palate, uvula, conjunctiva, skin, larynx, trachea, bronchus, scalp, penis and vagina. The causative organism is Rhinosporidium Seebri, first described in 1900 by Guyllermo Seebri ${ }^{[1,2]}$, Majority of cases are reported in India and Sri Lanka. Mode of spread is from dung of infected cattle, stagnant water sources like ponds and tanks in endemic areas.

Rhinosporidium is usually a result of direct traumatic inoculation with the organism. 
Trauma from Surgery has also been cited as potential mode of transmission ${ }^{[1,3,4]}$.

This disease is commonly seen in adult men with male female ratio of $3: 1-4: 1$ Few attainable datas shows higher incidence in subject with blood group $O$. The presumed mode of infection from the natural aquatic habitat of R. seebri is through traumatized epithelium most commonly in nasal cavities. In the nasal cavity septum, inferior turbinate, inferior meatus, floor are commonly involved.

Characterestically, Rhinosporidial lesion in nasal cavity are polypoidal, granular and red in colour with the surfaces studded with sporangia. Spillage of endospores from Rhinosporidiosis mass during surgery leads to autoinoculation to adjacent epithelium causing multiple polyp in recurrent diseases ${ }^{[5]}$. There is evidence of spread to anatomically distant sites such as limbs, trunk by development of subcutaneous granulomas without breach of overlying skin ${ }^{(6)}$. Rhinosporidiosis also affects bulbar and palpebral conjunctiva. Rhinosporidiosis of lacrimal sac and nasolacrimal duct has also been well documented probably due to autoinoculation from conjunctiva.

Most cases present with epistaxis and nasal obstruction. Rhinosporidiosis is rarely fatal. Hemorrhagic and disseminated Rhinosporidiosis otherwise called malignant Rhinosporidiosis may lead to death. The most successful treatment reported is surgical excision with cauterization of base by diathermy.

\section{Materials and Methods}

It was a prospective study carried out from Jan 2016 - June 2018. This series included 40 subjects with a clinical and histopathological diagnosis of Rhinosporidiosis treated at Kanyakumari Government Medical College.

A detailed history including age, sex, duration of symptoms, area of residence and interval of recurrence were analysed. Particular focus was placed on bathing habits. Clinical examination, diagnostic nasal endoscopy and video laryngoscopy were done to localize the site and extent of lesions in each case. All cases were provisionally diagnosed on a clinical basis. History of other medical or surgical illness was also recorded. All routine hematological investigation including blood group of each patient were also done. All subjects were treated by wide surgical excision and electrocautery of the base of the lesion with the aid of endoscope and appropriate instrument under general anesthesia. Specimen was sent for histopathological examination.

\section{Observation and Results}

A total of 40 cases were treated over a period of $2^{1 / 2}$ years (January 2016 - June 2018). There were 32 males $(80 \%)$ and 8 females $(20 \%)$ in the study. Age distribution varies from 22 years to 72 years with predominance in 41-50 years age group. Among males 41 to 50 is the common age group while in females $31-40$ is the common age group.

Table 1 : Age - Sex Distribution

\begin{tabular}{|l|c|c|}
\hline Age & Male & Female \\
\hline $20-30$ & 2 & 2 \\
\hline $31-40$ & 3 & 3 \\
\hline $41-50$ & 6 & 1 \\
\hline $51-60$ & 4 & 1 \\
\hline $61-70$ & 2 & 1 \\
\hline$>70$ & 1 & 0 \\
\hline
\end{tabular}

Commonest presentation is epistaxis (40\%) followed by nasal obstruction (40\%), anosmia (16\%), Dysphagia (5\%) Dyspnoea (2\%).

Table 2: Presentation of recurrent Rhinosporidiois

\begin{tabular}{|l|c|}
\hline Symptoms & Percentage \\
\hline Epistaxis & $40 \%$ \\
\hline Nasal obstruction & $40 \%$ \\
\hline Anosmia & $16 \%$ \\
\hline Dysphagia & $5 \%$ \\
\hline Dyspnoea & $2 \%$ \\
\hline
\end{tabular}

Among the 40 cases $98 \%$ have multiple attachments whereas one case has tracheal attachment. 
Commonest site is both Nasal cavities 17 $(42.5 \%)$

Naso - Nasopharynx 10 (25\%), lacrimal sac 5 (12.5 \%), Oropharynx $4 \quad(10 \%)$, Laryngopharynx 2 (5\%), Trachea 1 (2.5\%) and Disseminated Cutaneous Rhinosporidiois 1 $(2.5 \%)$

Table -3

\begin{tabular}{|l|c|}
\hline Site of Origin & Percentage \\
\hline Both Nasal Cavities & $42.5 \%$ \\
\hline Naso - Nasopharynx & $25 \%$ \\
\hline Lacrimal Sac & $12.5 \%$ \\
\hline Oropharynx & $10 \%$ \\
\hline Laryngopharynx & $5 \%$ \\
\hline Trachea & $2.5 \%$ \\
\hline Cutaneous & $2.5 \% \mathrm{~s}$ \\
\hline
\end{tabular}

\section{Pre -Op}



\section{Post - Op}

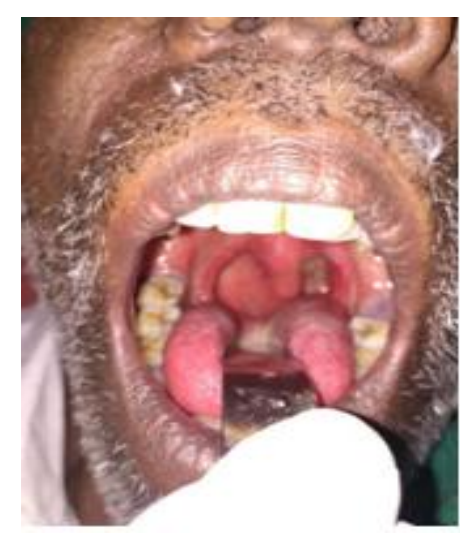

\section{Rhinosporidiois mass}



\section{Discussion}

Rhinosporidiosis is disease of low incidence, limited to the endemic zones in tropical and subtropical regions of India and Sri Lanka ${ }^{[1,2]}$. Literature review revealed little about the possible habitat and mechanism of transmission of this pathogen. The most accepted hypotheses are pointing to a relation contact with Stagnant water $^{[5]}$. Mode of spread is from dung of infected cattle. Direct Animal-to-man transmission has not been proven. In our series, all subjects had a history of taking bath in ponds. It is suggested that the contact of the nasal mucosa with contaminated water inoculated through microtraumas is a possible method of transmission that explains the predominance of lesions in the nasal cavities. The populations of low economic status are more frequently affected, with the majority of patients coming from agricultural zones, and those taking baths in ponds ${ }^{[7]}$

Recurrent cases may occur between the ages of 21 to 70 years with a maximum incidence between the ages of 41 and 50 years. Rhinosporidiosis is more common in males than females. Although the sex distribution varies in different reports, this may be due to the fact that males more typically have jobs related to land and backwater ${ }^{[8]}$. We did observe a male predominance in relation to the sex $(3: 1)$. Most of the cases observed in our series were between 41 and 50 years of age.

Soft and friable polypoidal mass studded with spores can be found upon examination. We observed a similar pattern in appearance of the lesions in our series and found it to be an important diagnostic criterion as far as rhinosporidiosis is concerned ${ }^{[7]}$. Significantly all the cases of recurrent Rhinosporidiosis showed multiple attachment.

The most frequent site is the nasal cavity although it is found in other localizations such as the nasopharynx, larynx, trachea. It can affect more than one site. The most common nasal sites are in decreasing order, nasal cavities, 
nasopharynx, Laryngopharynx, trachea and skin. Our findings suggest that the nasal cavity was the common site of involvement. $42.5 \%$ cases had bilateral involvement and $25 \%$ cases had nasopharynx involvement and $10 \%$ had oropharynx attachment. The progression of symptoms is nasal blockage, epistaxis, Anosmia dysphagia and dyspnea. Clinically it presents as a polyploidy mass which is friable, painless, sessile or peduncle and finely lobated with small yellow points over the mass. corresponding to mature sporangia. Our findings revealed Masses in nasopharynx and oropharynx pedunculated with patient presenting with hotpotato voice. Nasal obstruction and epistaxis, being the most common presenting symptom. The diagnosis is based on clinical history, detailed otorhinolaryngological examination with the help of Diagnostic nasal endoscopy and video laryngoscopy.

\section{Conclusion}

Rhinosporidiosis is disease of dubious etiology affecting mainly farmers and is common in tropical regions of the world. Wide local excision with cauterization of base is the main modality of treatment. Almost all recurrent cases have multiple attachment probably as a result of direct traumatic inoculation with the organism during previous surgery. Extra nasal spread is very common in recurrent cases. Excision with Diathermy cauterization of base is the treatment of choice. Special care in management of primary Rhinosporidiosis helps in prevention of extra nasal spread.

\section{Acknowledgement}

I would like to thank all my patients, who were subjected in this study. I would like to thank my wife Dr. D.S. Bebincy M.D; DGO for her constant encouragement and support.
Funding: No funding sources

Conflict of interest: None declared

Ethical approval: Nor required

\section{Reference}

1. Arseculeratne SN. Recent advances in Rhinospordiosis and Rhinospordium seeberi. Indian J. Med. Microbiology 2002, 20 (19 13)

2. Ayub ur- Rehman, Muhammad MN, Rhinospordiosis

3. Rajeswari A, Gangadhala. S etal Rhinospordiosis - a report of tow cases, Indian J otolaryngol head and Neck surgery 2010, 62(3) $322-325$.

4. Mathew J, Pandhys etal Tele laryngoscopy guided flexible fibreoptic intubation for laryngeal Rhinospordiosis. Int Anaesthesia Res sec. 2010, 110 (4) 1066 -1068.

5. Karunaratne wne : Rhinospordiosis in man 1964 . The Altolone press, London.

6. Rajan RV, Viswanathan GC, Rhinospordiosis, A Study with a report of a fatal case with systemic dissemination. Indian J surg, 1955, $17: 269-298$.

7. Sajilal Manonmony, Regee Ebenezer, Renjit etal, Rhinospordiosis, International journal of Biomedical research 2015, 6 (06) : 416 - 420

8. Palashkumar mandal, Disseminated cutaneous Rhinospordiosis, a tumor like lesion with Therapeutic challenge, a case report. Iranian journal of pathology, Vol :9, No : 4, fall 2014. 